\title{
CIRED 2021 - 26. Internationale Konferenz über elektrische Verteilnetze
}

\author{
H. Struber OVE
}

angenommen am 19. Oktober 2021, online publiziert am 12. November 2021 (c) Springer-Verlag GmbH Austria, ein Teil von Springer Nature 2021

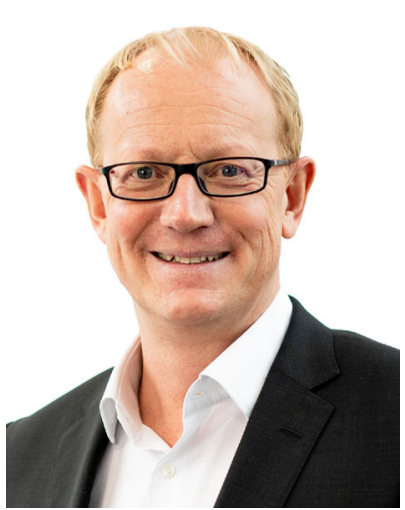

Dipl.-Ing. Herwig Struber, MSc
Alle zwei Jahre findet die internationale Konferenz der CIRED über elektrische Verteilnetze statt. Die Konferenz bietet eine ausgezeichnete Plattform zu allen technischen Fragen in Verteilnetzen, dem aktuellen Stand der Technik sowie laufenden Forschungs-, Innovationsund Entwicklungstätigkeiten. Neben dem technischen Schwerpunkt umfasst die CIRED den gesamten Bereich der Stromverteilungssysteme und der damit verbundenen Dienstleistungen, einschließlich der Fragen der verteilten Stromerzeugung und neuesten Entwicklungen.

Die CIRED bildet somit eine exzellente Gelegenheit für all jene, die sich als Teil der Stromverteilungscommunity verstehen, unabhängig davon, ob sie aus dem Verteilungs-, Erzeugungs-, Beratungs-, Dienstleistungs- oder aus dem akademischen Sektor stammen.

Nach 2019 in Madrid hätte die 26. CIRED-Konferenz vom 20.-23. September 2021 in Genf stattfinden sollen. Obwohl zu Beginn des Jahres noch Zuversicht herrschte, im September die Konferenz in Genf durchführen zu können, musste man aufgrund der anhaltenden Unsicherheiten im Zusammenhang mit der COVID-19-Pandemie letztlich im Mai 2021 die Entscheidung treffen, auf eine vollständig virtuelle Konferenz umzusteigen. Die Gesundheit und Sicherheit aller Teilnehmer/innen hatten bei dieser Entscheidung oberste Priorität. Durch diese frühe Entscheidung hatte man noch hinreichend Zeit, den Wechsel auf eine virtuelle Konferenz zu organisieren und eine qualitativ hochwertige Konferenz für alle Teilnehmer/innen zu bieten.

Die 26. CIRED-Konferenz 2021 wurde somit erstmals in rein virtueller Form abgehalten, 941 Teilnehmer/innen nutzten die vier Tage zum umfangreichen Wissens- und Erfahrungsaustausch. Aus Österreich waren 46 Teilnehmer/innen gemeldet. Aus den mehr als 1.000 zur Konferenz eingereichten Beiträgen wurden in einem umfassenden Evaluierungsprozess, in den auch zahlreiche österreichische Expert/innen eingebunden waren, die interessantesten Beiträge für die Konferenz ausgewählt.

Zusammengefasst kann man sagen, dass der Wechsel in eine virtuelle Umgebung ebenso viele Möglichkeiten wie die bisherigen Konferenzen bot, Forschungsergebnisse auszutauschen, hochwertige Fachbeiträge zu diskutieren und Networking zu betreiben, die CIRED letztlich so bekannt und erfolgreich gemacht haben.

Mit 24 österreichischen Beiträgen blieb die Anzahl, verglichen mit den letzten Konferenzen, auf einem sehr hohen Niveau. Sehr erfreulich aus österreichischer Sicht war auch, dass Frau Nina Fuchs (AIT) stellvertretend mit dem Paper 450 "Simulation Tool for the TechnoEconomic Analysis of Hybrid AC/DC Low-Voltage Distribution Grids" mit dem „Best Young Academic Paper Award - CIRED 2021" prämiert wurde.

Die fachliche Gliederung der Konferenz in sechs Bereiche (Sessions) wurde wie auch schon in den letzten CIRED-Konferenzen aufrechterhalten:

1) Network Components

2) Power Quality and Electromagnetic Compatibility

3) Operation, Control, and Protection

4) Distributed Energy Resources and Efficient Utilization of Electricity

5) Planning of Power Distribution Systems

6) DSO Customers, Regulation, and Business Models

Die Konferenz wurde auch virtuell nach dem bekannten CIREDAblauf abgehalten. Nach der offiziellen Eröffnung und den Tutorials am Montag wurden an den folgenden drei Konferenztagen die Beiträge der einzelnen Sessions präsentiert. Die Beiträge wurden in den Main-Sessions, Round Table-Diskussionen und moderierten PosterSessions vorgestellt.

Im Anschluss an die virtuelle Konferenz fand die Sitzung des ClRED Directing Committees statt. Neben einer ersten Analyse zur CIRED-Konferenz 2021 standen die Planung des CIRED-Workshops in Portugal 2022 sowie der nächsten CIRED-Konferenz 2023, welche in Rom stattfinden wird, im Fokus.

Das österreichische Nationalkomitee CIRED bedankt sich an dieser Stelle bei allen österreichischen Kolleg/innen, welche in der Vorbereitung und/oder auch während der Konferenz ihren wertvollen Beitrag einbrachten.

Für diejenigen, die an der virtuellen Konferenz nicht teilnehmen konnten, bietet das österreichische Nationalkomitee CIRED wieder zwei Infonachmittage mit Präsentationen über die aktuellen Trends der sechs Sessions an:

CIRED-Infonachmittag des österr. Nationalkomitees Wien, Dienstag, 25. Jänner 2022

In den Räumlichkeiten des OVE, Eschenbachgasse 9 1010 Wien

CIRED-Infonachmittag des österr. Nationalkomitees Innsbruck, Dienstag, 01. März 2022

AC Hotel Innsbruck, Salurner Straße 15

6020 Innsbruck

Die österreichischen CIRED-Veranstaltungen sind auch im OVEVeranstaltungskalender angeführt.

Struber, Herwig, Salzburg Netz GmbH, Bayerhamerstraße 16, 5020 Salzburg, Österreich (E-Mail: herwig.struber@salzburgnetz.at) 
Weitere CIRED-Veranstaltungen sind:

ETG-CIRED-(D-A-CH)-Workshop 2021 - München, Deutschland 01.-02. November 2021, Hanns-Seidel-Stiftung e.V.

Thema: Innovationen im Verteilnetz

CIRED-Workshop 2022 - Porto, Portugal

02.-03. Juni 2022 Thema: E-mobility and power distribution systems

CIRED-Konferenz 2023 - Rom, Italien

12.-15. Juni 2023

Die nachfolgenden Beiträge über die CIRED-Sessions stellen Kurzfassungen der Inhalte und Trends dar und sollen zu einer intensiveren Befassung mit den Beiträgen der CIRED-Konferenz anregen. Besten Dank auch an die österreichischen Berichterstatter!
Weiterführende Informationen oder Ansprechpartner finden Sie auf den folgenden Websites:

http://www.cired.at

http://www.cired.net

http://www.cired2021.org

https://www.cired2022porto.org

http://wwwcired2023.org

Hinweis des Verlags Der Verlag bleibt in Hinblick auf geografische Zuordnungen und Gebietsbezeichnungen in veröffentlichten Karten und Institutsadressen neutral. 